\title{
Prognostic role of upregulated P300 expression in human cancers: A clinical study of synovial sarcoma and a meta-analysis
}

\author{
ZIHAN LIU ${ }^{1 *}$, YONGLAI HE ${ }^{2 *}$, XIAOJUAN LIAN ${ }^{3 *}$, HONG ZOU $^{2}$, YALAN HUANG $^{2}$, NING WANG $^{2}$, \\ JIANMING HU ${ }^{2}$, XIAOBIN CUI ${ }^{2}$, JIN ZHAO ${ }^{2}$, WENJIE ZHANG ${ }^{2}$, WENYI GU ${ }^{4}$, LIJUAN PANG ${ }^{2}$ and YAN QI ${ }^{2}$ \\ ${ }^{1}$ Department of Pathology, Shihezi University School of Medicine and The First Affiliated Hospital to Shihezi University \\ School of Medicine, Shihezi, Xinjiang 832002; ${ }^{2}$ Department of Emergency, Jinshan Branch Shanghai Sixth People's \\ Hospital Affiliated to Shanghai Jiaotong University, Shanghai 200233; ${ }^{3}$ Department of Blood Cancers, Jiangjin \\ Central Hosptial of Chongqing, Chongqing 400042, P.R. China; ${ }^{4}$ Australian Institute for Bioengineering and \\ Nanotechnology, University of Queensland, Brisbane, QLD 4072, Australia
}

Received April 20, 2018; Accepted March 21, 2019

DOI: $10.3892 /$ etm.2019.7906

\begin{abstract}
E1A binding protein p300 (P300) is a member of the histone acetyltransferase family of transcriptional co-activators, which are associated with various types of cancer. Numerous studies have evaluated the diagnostic value of P300, but their results are not consistent. Therefore, a clinical study and a meta-analysis were performed in the present study to investigate the prognostic value of P300 expression in human malignant neoplasms. Immunohistochemical (IHC) analysis was used to assess P300 expression in 43 paraffin-embedded primary synovial sarcoma (SS) samples. For the meta-analysis, eligible studies published until January 21, 2018 were identified by searching the PubMed, EMBASE and Web of Science databases. The IHC analysis indicated a high P300 expression rate in $33.3 \%(10 / 30)$ of biphasic SS (BSSs) and in 60\% (6/10) of monophasic fibrous SS tissues. In BSS, the expression rate was significantly higher in the epithelial component $(80.0 \%, 24 / 30)$ than that in the spindle-cell component $(30.0 \%, 9 / 30 ; \mathrm{P}<0.05)$. The meta-analysis indicated that high expression of P300 was associated with poor overall survival (OS) in digestive system malignant neoplasms ( $\mathrm{HR}=1.54,95 \% \mathrm{CI}$ : $1.20-2.23)$, as well as with poor progression-free survival, recurrence-free survival and disease-free survival combined $(\mathrm{HR}=1.84,95 \% \mathrm{CI}$ : 1.36-2.47). Analysis of subgroups by ethnicity demonstrated that high expression of $\mathrm{P} 300$ was associated with poor OS in
\end{abstract}

Correspondence to: Dr Lijuan Pang or Dr Yan Qi, Department of Pathology, Shihezi University School of Medicine and The First Affiliated Hospital to Shihezi University School of Medicine, 59 North 2nd Road, Shihezi, Xinjiang 832002, P.R. China

E-mail: ocean123456@163.com

E-mail: qiyanyan-1998@163.com

\section{*Contributed equally}

Key words: P300, malignant neoplasm, clinical study, meta-analysis, prognosis, survival
Asians (HR=1.72, 95\% CI: 1.20-2.47) but favourable OS in Caucasians (HR=0.59, 95\% CI: 0.47-0.73). Furthermore, high expression of P300 was associated with clinical stage [Relative Risk (RR) $=1.30,95 \%$ CI: 1.07-1.58], lymph node metastasis $(\mathrm{RR}=1.30,95 \% \mathrm{CI}: 1.03-1.64)$ and depth of invasion $(\mathrm{RR}=1.31$, 95\% CI: 1.07-1.60). P300 expression may therefore be a useful biomarker for predicting patient prognosis in various types of human cancer.

\section{Introduction}

E1A binding protein P300 (P300; also known as KAT3B) is a member of the histone acetyltransferase family of transcriptional co-activators. It has a variety of roles in the transcription process and catalyzes histone acetylation through exerting its histone acetyltransferase activity $(1,2)$. It is involved in various cellular processes, including DNA damage response, cell-cycle regulation and proliferation, differentiation and apoptosis (3).

Previous studies have indicated that P300 has a tumour suppressor role in certain human cancer types, including colorectal cancer (CRC) and gastric cancer (4). Wang et al (5), reported that octreotide inhibits the proliferation of gastric cancer cells through P300 histone acetyltransferase activation and the interaction of zinc-activated ion channels and P300. As P300 binds to and inactivates adenovirus E1A, it has been considered a tumour suppressor (6). At the same time, P300 activates p53 to inhibit the proliferation of cancer cells (7). However, various studies have indicated that P300 is a positive regulator of cancer progression and is involved in tumorigenesis. Li et al (8) reported that overexpression of P300 is associated with aggressive features and poor prognosis in hepatocellular carcinoma (HCC). Isharwal et al (9), suggested that P300 may serve as a biomarker to predict prostate cancer recurrence and is associated with changes in the size and shape of epithelial cell nuclei. Xiao et al (10), revealed that high expression of P300 in breast cancer is associated with tumour recurrence and poor prognosis. Chen et al (11), determined that high P300 expression is associated with unfavourable survival outcomes in laryngeal squamous cell carcinoma (LSCC) patients. Although it has been diffusely reported, 
the prognostic value of P300 in various human cancer types remains controversial. Therefore, a clinical study of synovial sarcoma (SS) tissue samples and a meta-analysis were herein performed to evaluate the diagnostic and prognostic value of P300. The aim of the present study was to systematically investigate whether a high level of P300 expression may be used as a diagnostic and/or prognostic marker for cancer, and whether P300 may serve as a target for developing more effective therapies.

In the present two-fold study, SS samples were analysed in a clinical study, and data from previous studies from various countries were collected for a meta-analysis in order to assess the prognostic value of $\mathrm{P} 300$ expression in cancer patients.

\section{Materials and methods}

Specimens and clinicopathological characteristics. The present study included retrospectively collected paraffin-embedded samples from 43 patients with SS, including 30 biphasic SS (BSS), 10 monophasic fibrous SS (MFSS) and 3 poorly-differentiated SS (PDSS). These patients were treated at the First Affiliated Hospital of Shihezi University School of Medicine and the First Affiliated Hospital of Xinjiang Medical University (Shihezi, China) between January 1968 and December 2015. SS was diagnosed via histological and immunohistochemical (IHC) analyses by two senior pathologists. The histopathological grading of SS was based on the FNCLCC guidelines (12) for soft-tissue tumors. The present study was approved by the Internal Ethics Review Board of Shihezi University School of Medicine (Shihezi, China).

IHC staining and scoring. IHC staining was performed with an Envision two step kit (cat. no. PV-9003; Beijing Zhongshang Jinqiao Biotechnology Co., Ltd.) according to the manufacturer's protocols. The following antibodies were used: P300 (dilution, 1:500; cat. no. ab54984; Abcam), $\beta$-catenin (dilution, 1:400; cat. no. ab32572; Abcam). The scoring evaluation of $\beta$-catenin was performed as in a previous study by our group (12). Cells with nuclear staining were considered positive for P300. The P300 IHC scoring criteria were as follows: $0-1,<5 \%$ positive tumour cells or no staining; $2-4,6-25 \%$ positive tumour cells and light-brown nuclei; 5-8, 26-50\% positive tumor cells and brownish yellow nuclei; $9-12,51-75 \%$ positive tumor cells and brown nuclei.

Statistical analysis. SPSS17.0 software (SPSS, Inc.) was used for statistical analysis of the results of the above clinical study, with the chi-square test or Fisher's exact test used to compare differences between groups. $\mathrm{P}<0.05$ was considered to indicate a statistically significant difference.

Publication search for the meta-analysis and inclusion and exclusion criteria. For the meta-analysis, we the PubMed, EMBASE and Web of Science databases were searched for entries up to 21 January 2018 to identify relevant studies. Several combinations of the following keywords were applied: 'cancer', 'carcinoma', 'neoplasm', 'tumour', 'P300', 'survival', 'prognosis', 'metastasis' and 'sarcoma'. Studies were considered eligible if they met all of the following criteria: i) Patients with any malignant neoplasm [nasopharyngeal carcinoma
(NPC), non-small cell lung cancer (NSCLC), LSCC, SCLC, oesophageal squamous cell carcinoma (OSCC), HCC, CRC, gastroesophageal junction cancer melanoma (GEJC), cutaneous squamous cell carcinoma (CSCC) and breast cancer]; ii) investigation of the association between $\mathrm{P} 300$ expression and prognostic factors; iii) Kaplan-Meier survival analysis of 5-year overall survival; and iv) full-text articles written in English. Articles were excluded based on the following criteria: i) Review articles, laboratory studies or letters; ii) results that overlapped with or were duplicates of previously reported data; iii) studies lacking the key data of hazard ratios (HRs) or confidence intervals (CIs) without survival curves.

Data extraction and quality assessment. The data extracted mainly included the following: i) First author name and publication year; ii) nationality, ethnicity and size of the cohort, and type of clinical disease; iii) clinical stages, lymph node metastasis, depth of invasion, sex, tumour size and degree of differentiation; iv) HRs of elevated P300 expression regarding overall survival (OS), progression-free survival (PFS), recurrence-free survival (RFS) and disease-free survival (DFS), along with their 95\% CIs and P-values. If HRs and 95\% CIs were not directly reported in articles, and only Kaplan-Meier survival data were available, the data were extracted from the graphical survival plots to estimate HRs and 95\% CIs. The information was carefully and independently extracted by two authors (YL and $\mathrm{ZH}$ ) according to the critical review checklist of the Dutch Cochrane Centre proposed by Meta-Analysis Of Observational Studies In Epidemiology (MOOSE) (13). In cases of disagreement, a consensus was reached by discussion.

Subgroup meta-analysis and influence of clinicopathological factors. In addition to analyzing the association between P300 expression and survival, subgroup analyses by ethnicity (Asian vs. Caucasian) and cancer location were performed. The association between P300 expression and clinicopathological factors, including sex, tumour size, grade of differentiation, clinical stage, lymph node metastasis and depth of invasion was also analysed.

Statistical methods for the meta-analysis. HRs and 95\% CIs were calculated using Cochran's Q test and the Higgins I-squared statistics. $\mathrm{P}<0.05$ was considered to indicate statistical significance, and $\mathrm{I}^{2}>50 \%$ was considered to indicate heterogeneity. A random-effects model (Der Simonian and Laird method) was applied. Otherwise, the fixed-effects model (Mantel-Haenszel test) was used. In addition, stratified analyses were used to minimize the influence of heterogeneity. Publication bias was estimated via Egger' test with a funnel plot (14). All statistical analyses were performed using STATA software (version 12.0; Stata Corp.).

\section{Results}

Clinical study population. The sex ratio of patients was 1.04:1 (22 males and 21 females), and the age at diagnosis ranged from 10 to 76 years (mean, 39 years). The tumours were widely distributed; however, most arose in the extremities $(n=24$, $55.8 \%)$ and trunk $(n=12,27.9 \%)$. A total of 5 tumours $(11.6 \%)$ arose in the head and neck and $2(4.7 \%)$ in the pelvis/peritoneum (data not shown). 
Table I. Differential expression of P300 proteins between BSS and MFSS.

\begin{tabular}{lrrrr}
\hline & \multicolumn{3}{c}{ P300 (n) } & \\
\cline { 2 - 3 } Histological type & - & + & $++/+++$ & P-value \\
\hline BSS & 2 & 18 & 10 & $0.304^{\text {a }}$ \\
MFSS & 0 & 4 & 6 & \\
Epithelioid cells & 1 & 5 & 24 & $<0.001^{\text {b }}$ \\
Spindle cells & 3 & 18 & 9 & \\
\hline
\end{tabular}

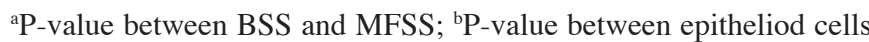
and spindle cells. BSS, biphasic synovial sarcomas; MFSS, monophasic fibrous synovial sarcomas; P300, E1A binding protein P300.

IHC analysis of P300 expression in BSS and MFSS. The IHC staining results are presented in Table I and representative IHC images are provided in Fig. 1. P300 expression was present in BSS $(28 / 30,93.3 \%)$ and MFSS (10/10, $100 \%$ ). Strongly positive P300 expression was noted in 10/30 (33.3\%) BSS samples and 6/10 (60\%) MFSS samples. Although the rate of strongly positive P300 expression in MFSS was higher than that in BSS, this difference was not statistically significant ( $\mathrm{P}>0.05$; Table I; Fig. $1 \mathrm{~A}$ and $\mathrm{B})$. In addition, immunohistochemical expression of $\mathrm{P} 300$ proteins were markedly different between the epithelioid and spindle cell components of BSS. Furthermore, P300 expression was present in epithelial cells $(29 / 30 ; 96.7 \%)$ and spindle cells (27/30; 90\%). Strongly positive P300 staining was observed in 24/30 (80\%) of epithelioid cells and 9/30 (30\%) of spindle cells, and the rate of strongly positive P300 expression was significantly higher in epithelioid cells $(\mathrm{P}<0.05$; Table I; Fig. 1C and D).

Meta-analysis study selection and characteristics. Initially, a total of 161 potentially relevant articles were identified from the literature search, but 126 articles were excluded due to being meeting abstracts $(n=7)$, research articles on cell lines $(n=89)$ or animal models $(n=10)$, or not being associated with prognosis $(n=20)$. After reading the full text of the studies selected, 21 articles were further excluded due to insufficient data on survival. Ultimately, 14 studies $(8,10,11,15-25)$ comprising 2,517 cases were included in the final meta-analysis (Fig. 2).

Among the 14 eligible studies included in the meta-analysis, 12 were on Asian populations (China, Korea, Japan) and two on Caucasian populations (British). The malignant neoplasms in the studies included CSCC, SCLC, SCC, GEJC, NPC, NSCLC, LSCC, HCC, OSCC, melanoma, CRC and breast cancer. Of the studies analysed, 4 focused on PFS/RFS/DFS and 13 reported on the OS of patients. The maximum follow-up period ranged from 50 to 250 months. The characteristics of the publications are provided in Table II.

Meta-analysis of P300 expression and OS. OS data were extracted from 13 studies. The characteristics of these publications are presented in Table III. A random-effects model was used to estimate the impact of P300 expression on OS with a pooled $\mathrm{HR}$ and its $95 \% \mathrm{CI}\left(\mathrm{P}<0.05, \mathrm{I}^{2}=86.2 \%\right)$. The results indicated that overexpression of P300 was not significantly associated with poor OS (HR=1.42, 95\% CI: 0.96-2.10; Fig. 3A).

A subgroup analysis by ethnicity indicated that in the Asian populations, overexpression of $\mathrm{P} 300$ was significantly associated with poor OS (HR=1.72, 95\% CI: 1.20-2.47; Fig. 3B); however, overexpression of $\mathrm{P} 300$ predicted a favourable $\mathrm{OS}$ in Caucasians (HR=0.59, 95\% CI: 0.47-0.73; Fig. 3B).

Furthermore, subgroup analyses by location of the malignant neoplasms were performed. The results indicated that P300 overexpression was associated with poor OS of patients with malignant neoplasms of the digestive system $(\mathrm{HR}=1.64$, 95\% CI: 1.20-2.23; Fig. 3C). In HCC, high P300 expression was associated with poor OS (HR=1.93, 95\% CI: 1.11-3.36; Fig. 3D). However, high P300 expression was associated with favourable OS in melanoma (HR=0.59, 95\% CI: 0.47-0.73; Fig. 3D).

Meta-analysis of P300 expression and PFS/RFS/DFS. Analysis of data extracted from three studies for PFS/RFS/DFS and meta-analysis using a fixed-effects model $(\mathrm{P}=0.644$, $\mathrm{I}^{2}=0.0 \%$ ) suggested that overexpression of $\mathrm{P} 300$ is associated with poor PFS, RFS and DFS (HR=1.84, 95\% CI: 1.36-2.47; Fig. 4; Table IV).

Meta-analysis of P300 expression and clinicopathological factors. P300 expression was associated with clinicopathological factors, including sex, tumour size, degree of differentiation, clinical stage, lymph node metastasis and depth of invasion (Table V). P300 overexpression was associated with clinical stage [III/IV vs. I/II, relative Risk (RR) $=1.30,95 \%$ CI: 1.07-1.58; Fig. 5A], lymph node metastasis (M1 vs. M0, $R R=1.30,95 \%$ CI: 1.03-1.64; Fig. 5B) and depth of invasion (T3/T4 vs. T1/T2, $\mathrm{RR}=1.31$, 95\% CI: 1.07-1.60) (Fig. 5C). However, it was not significantly associated with sex (RR=0.97, 95\% CI: 0.90-1.06), tumour size ( $\leq 5$ vs. $>5 \mathrm{~cm}, \mathrm{RR}=0.89,95 \% \mathrm{CI}$ : $0.67-1.19$ ) or degree of differentiation (well + moderate vs. poor, $\mathrm{RR}=0.86$, 95\% CI: 0.71-1.04; Table VI).

A further subgroup analysis by digestive and respiratory system malignant neoplasms indicated that overexpression of P300 was associated with lymph node metastasis of respiratory system malignant neoplasms (M1 vs. M0, RR=2.15, $95 \%$ CI: 1.57-2.95; Fig. 5D).

Publication bias. Publication bias for patient survival and tumour progression was assessed by funnel plots and Egger's tests. As expected, the funnel plots of P-values of the Egger's test were 0.102 for OS (Fig. 6A) and 0.184 for RFS/PFS/DFS (Fig. 6B), suggesting no publication bias.

\section{Discussion}

EP300 is an important factor in the transforming growth factor- $\beta$ signaling pathway. EP300 somatic mutations are significantly different between the epithelial and spindle cell components in SS, and are mainly located in the DNA-binding domain and the gene encoding histone $\mathrm{H} 3$ lysine acetyltransferases (26). The clinical analysis included in the present study indicated that the expression of $\mathrm{P} 300$ was significantly different between the epithelial and spindle cell components. In addition, 

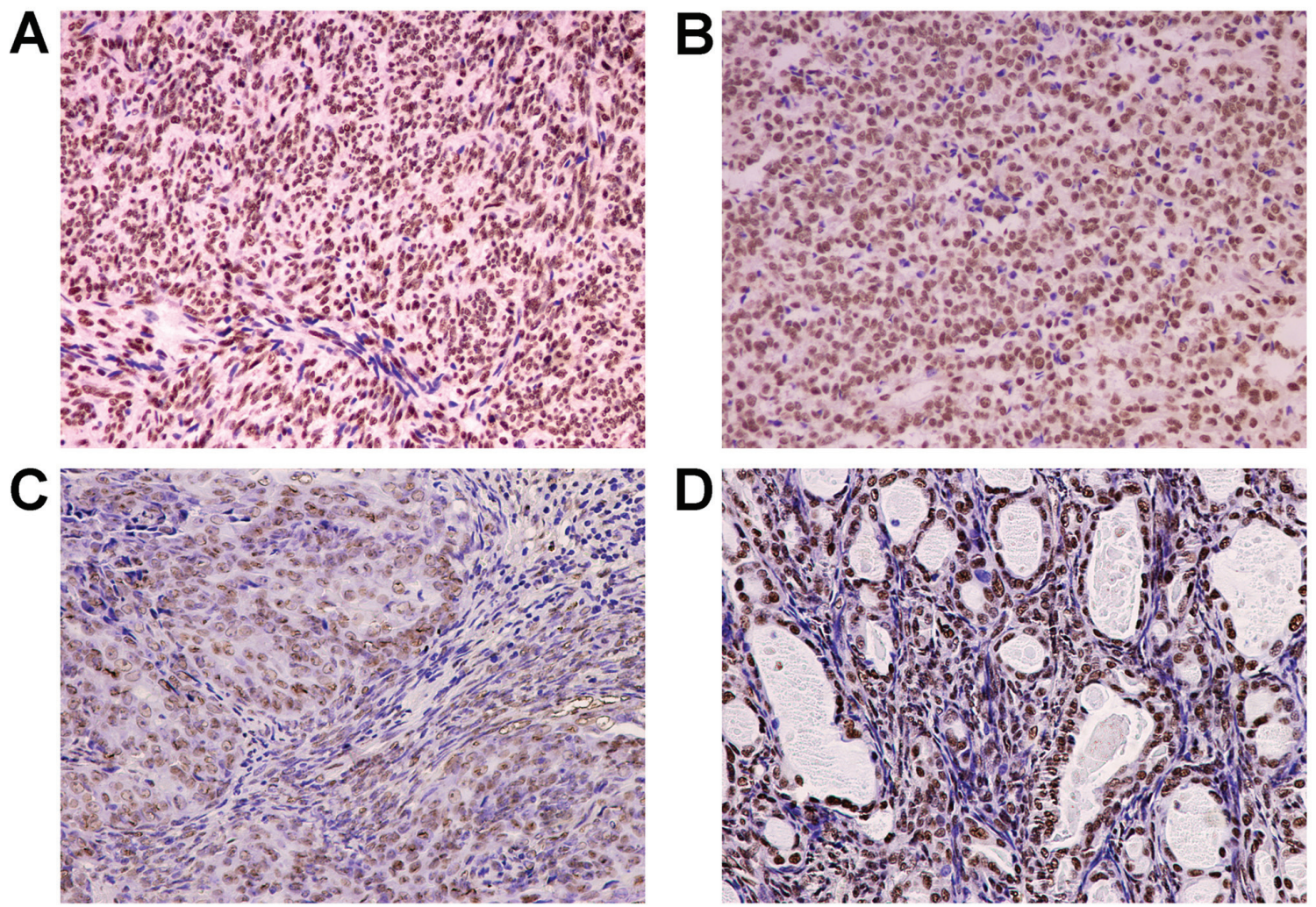

Figure 1. Immunohistochemical staining of different types of sarcoma tissue for P300 protein. (A) Monophasic fibrous synovial sarcoma and (B) poorly differentiated synovial sarcoma exhibited diffuse nuclear expression. (C and D) Biphasic synovial sarcoma samples frequently had nuclear expression of P300 in (C) nested epithelial cells and (D) glandular epithelium, and the P300 expression in epithelial components was higher than that in the surrounding spindle components (magnification, x200; brown staining indicates P300). P300, E1A binding protein P300.

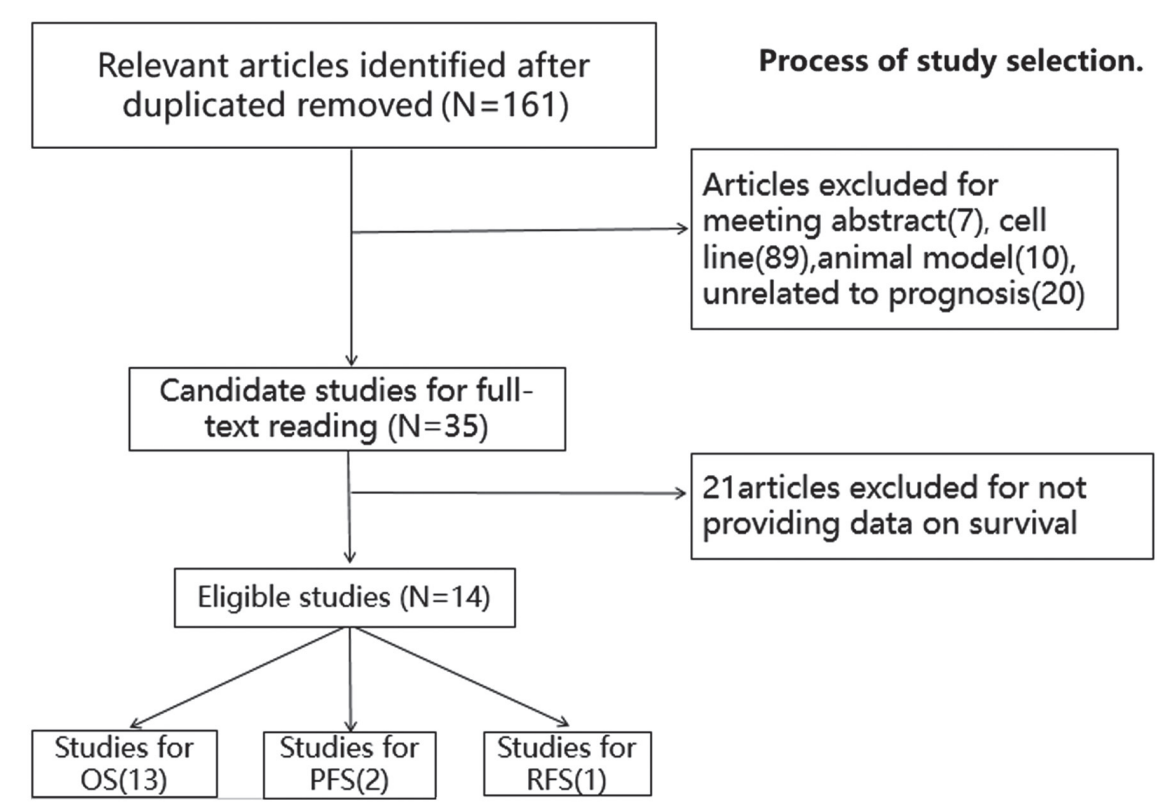

Figure 2. Flow chart depicting the process of study selection for the meta-analysis. OS, overall survival; PFS, progression-free survival; RFS, recurrence-free survival.

the association of P300 with epithelial-mesenchymal transition (EMT)-associated proteins that were assessed in a previous study by our group (12) were analyzed, and it was revealed that the expression of P300 was closely associated with the expression of Slug (Both P300 and slug were expressed in single-phase fibrous synovial sarcoma, and the association between the two was analyzed), suggesting that P300 may have an important role in the pathogenesis of SS (data not shown).

The present study further evaluated the prognostic role of upregulated P300 expression in SS with a comprehensive and 


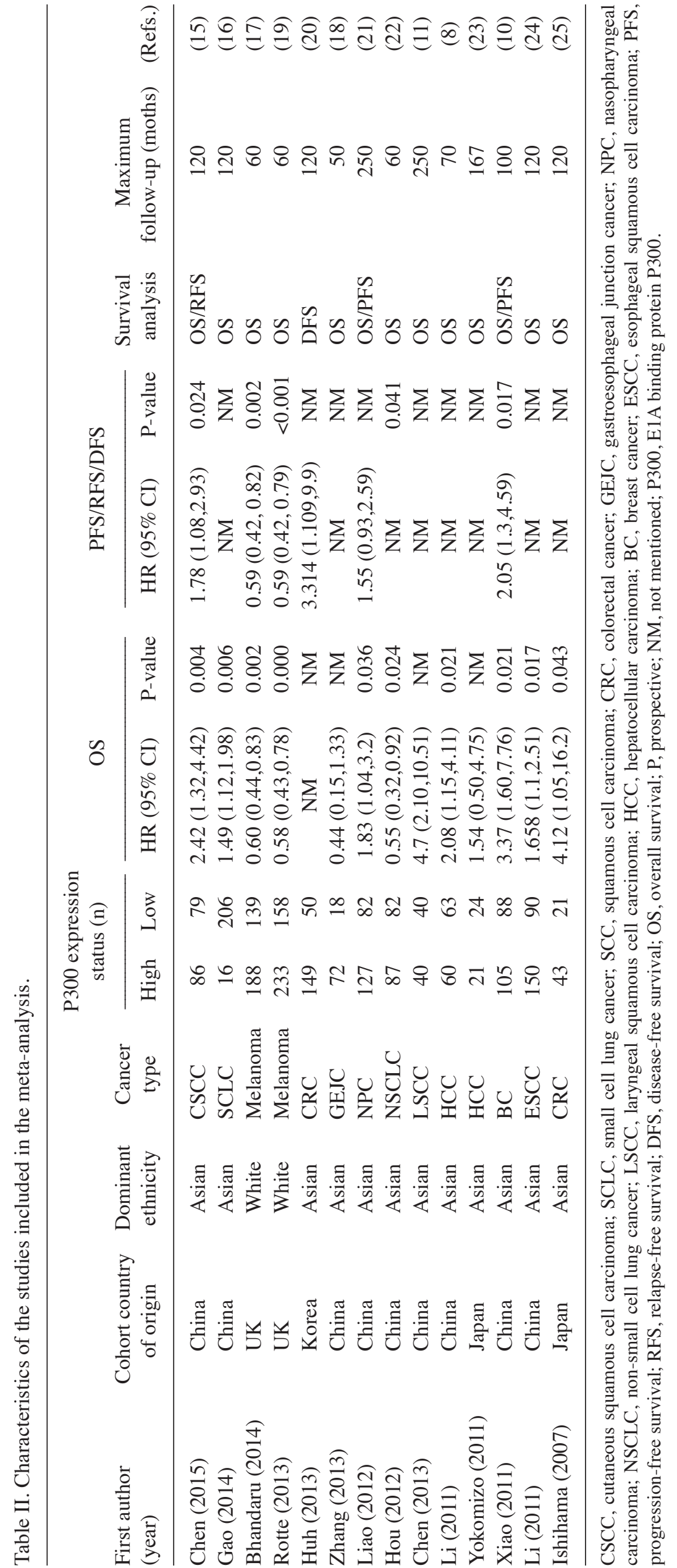




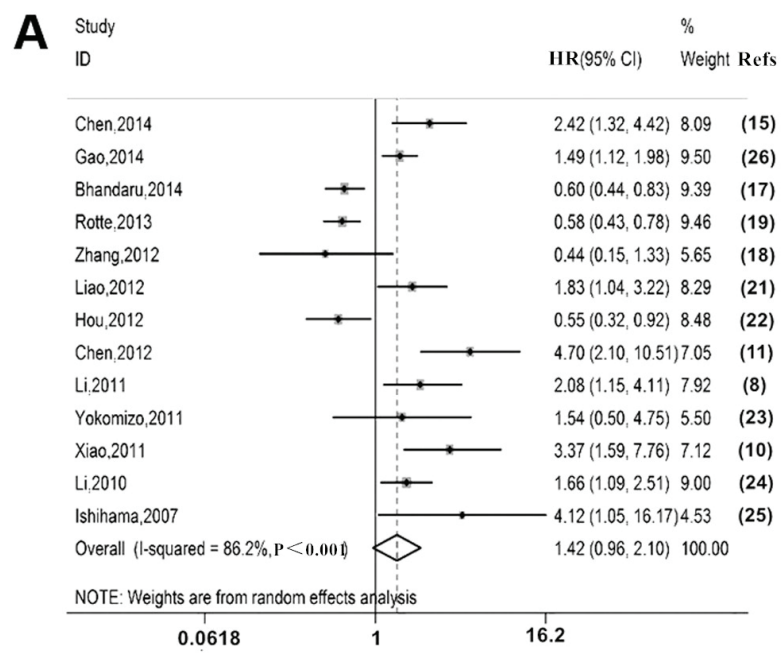

B ID Asian
Chen,2014 Gao,2014 Zhang, 2012 Liao,2012 Hou,2012 Chen 2012 Li 2011 Yi: 2011 Yokomizo,2011
Xiao:2011 Li, 2010 Ishihama,2007 Subtotal $($ I-squared $=72.6 \%, p=0.000)$

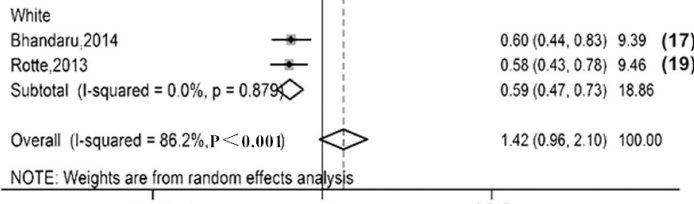
NOTE: Weights are from random effects analys

$$
\begin{array}{lll}
0.0618 & 1 & 16.2
\end{array}
$$

D

Study

ID

HR $(95 \%$ Cl) Weight Refs

$2.42(1.32,4.42) 8.09(15)$ $1.49(1.12 .1 .98) 9.50 \quad(26)$ $0.44(0.15,1.33) 5.65 \quad(18)$ $\begin{array}{lllll}1.83(1.04,3.22) & 8.29 & (21\end{array}$ $0.55(0.32,0.92) \quad 8.48 \quad(22)$ $4.70(2.10,10.51) 7.05 \quad$ (11) $208(1.15,411) 7.02$ $2.08(1.15,4.11) 7.92 \quad(8)$ $3.54(1.59,7.76) 7.12$ (10) $1.66(1.09,2.51) 9.00 \quad(24)$ $4.12(1.05,16.17) 4.53 \quad(25)$ $1.72(1.20,2.47) 81.14$

$0.60(0.44,0.83) 9.39 \quad(17)$ $0.58(0.43,0.78) 9.46 \quad(19)$ $0.59(0.47,0.73) 18.85$

C Digestive system

Study

ID

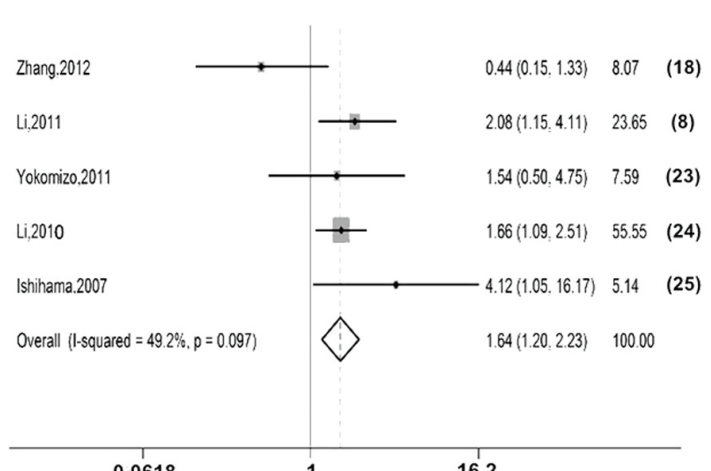

\section{rang}

melanoma

Bhandaru, 2014

Rotte,2013

Subtotal (I-squared $=0.0 \%, p=0.879$

HCC

Li,2011

Yokomizo,2011

Subtotal (I-squared $=0.0 \%, p=0.650$ )

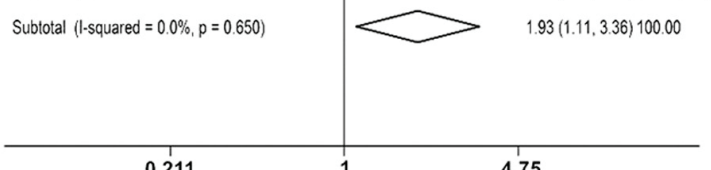

Figure 3. Forest plots assessing the association between E1A binding protein P300 expression and overall survival. (A) Total study population, (B) subgroup analysis by ethnicity (Asian and Caucasian), (C) The association between P300 expression and overall survival in digestive system tumors and (D) The association between P300 expression and overall survival in melanoma and HCC. The horizontal bar represents the confidence interval, the small square represents the HR and the diamon represents the overall HR with the confidence interval. HCC, hepatocellular carcinoma.

\begin{tabular}{|c|c|c|c|}
\hline Study & & $\%$ & \\
\hline ID & $\operatorname{HR}(95 \% \mathrm{Cl})$ & Weight & Refs \\
\hline chen,2014 & $1.78(1.08,2.93)$ & 35.80 & (15) \\
\hline Liao,2012 & $1.55(0.93,2.59)$ & 33.99 & (21) \\
\hline Xiao,2011 & $2.05(1.31,4.59)$ & 22.77 & $(10)$ \\
\hline Huh,2013 & $3.31(1.11,9.90)$ & 7.44 & (20) \\
\hline Overall $(I$-squared $=0.0 \%, p=0.644)$ & $1.84(1.36,2.47)$ & 100.00 & \\
\hline
\end{tabular}

Figure 4. Forest plot for the association between E1A binding protein P300 expression and tumour progression, progression-free survival, relapse-free survival and disease-free survival. The horizontal bar represents the confidence interval, the small square represents the HR and the diamon represents the overall HR with the confidence interval. 
Table III. Characteristics of the studies included in Fig 3.

\begin{tabular}{|c|c|c|c|c|c|c|c|c|c|c|}
\hline \multirow{2}{*}{$\begin{array}{l}\text { First author } \\
\text { (year) }\end{array}$} & \multirow{2}{*}{$\begin{array}{l}\text { Cohort } \\
\text { country } \\
\text { of origin }\end{array}$} & \multirow{2}{*}{$\begin{array}{l}\text { Dominant } \\
\text { ethnicity }\end{array}$} & \multirow{2}{*}{$\begin{array}{l}\text { Cancer } \\
\text { type }\end{array}$} & \multicolumn{2}{|c|}{$\begin{array}{c}\mathrm{P} 300 \\
\text { expression } \\
\text { status (n) }\end{array}$} & \multicolumn{2}{|l|}{ OS } & \multirow{2}{*}{$\begin{array}{l}\text { Survival } \\
\text { analysis }\end{array}$} & \multirow{2}{*}{$\begin{array}{c}\text { Maximum } \\
\text { follow-up } \\
\text { (moths) }\end{array}$} & \multirow[b]{2}{*}{ (Refs.) } \\
\hline & & & & High & Low & $\mathrm{HR}(95 \% \mathrm{CI})$ & P-value & & & \\
\hline Chen (2015) & China & Asian & CSCC & 86 & 79 & $2.42(1.32,4.42)$ & 0.004 & OS & 120 & $(15)$ \\
\hline Gao (2014) & China & Asian & SCLC & 16 & 206 & $1.49(1.12,1.98)$ & 0.006 & OS & 120 & (16) \\
\hline Bhandaru (2014) & UK & White & Melanoma & 188 & 139 & $0.60(0.44,0.83)$ & 0.002 & OS & 60 & $(17)$ \\
\hline Rotte (2013) & UK & White & Melanoma & 233 & 158 & $0.58(0.43,0.78)$ & 0.000 & OS & 60 & (19) \\
\hline Zhang (2013) & China & Asian & GEJC & 72 & 18 & $0.44(0.15,1.33)$ & NM & OS & 50 & (18) \\
\hline Liao (2012) & China & Asian & $\mathrm{NPC}$ & 127 & 82 & $1.83(1.04,3.2)$ & 0.036 & OS & 250 & $(21)$ \\
\hline Hou (2012) & China & Asian & NSCLC & 87 & 82 & $0.55(0.32,0.92)$ & 0.024 & OS & 60 & $(22)$ \\
\hline Chen (2013) & China & Asian & LSCC & 40 & 40 & $4.7(2.10,10.51)$ & NM & OS & 250 & $(11)$ \\
\hline Li (2011) & China & Asian & $\mathrm{HCC}$ & 60 & 63 & $2.08(1.15,4.11)$ & 0.021 & OS & 70 & (8) \\
\hline Yokomizo (2011) & Japan & Asian & $\mathrm{HCC}$ & 21 & 24 & $1.54(0.50,4.75)$ & NM & OS & 167 & $(23)$ \\
\hline Xiao (2011) & China & Asian & $\mathrm{BC}$ & 105 & 88 & $3.37(1.60,7.76)$ & 0.021 & OS & 100 & $(10)$ \\
\hline Li (2011) & China & Asian & ESCC & 150 & 90 & $1.658(1.1,2.51)$ & 0.017 & OS & 120 & $(24)$ \\
\hline Ishihama (2007) & Japan & Asian & $\mathrm{CRC}$ & 43 & 21 & $4.12(1.05,16.2)$ & 0.043 & OS & 120 & $(25)$ \\
\hline
\end{tabular}

CSCC, cutaneous squamous cell carcinoma; SCLC, small cell lung cancer; SCC, squamous cell carcinoma; CRC, colorectal cancer; GEJC, gastroesophageal junction cancer; NPC, nasopharyngeal Carcinoma; NSCLC, non-small cell lung cancer; LSCC, laryngeal squamous cell carcinoma; HCC, hepatocellular carcinoma; BC, breast cancer; ESCC, esophageal squamous cell carcinoma; OS, overall survival; NM, not mentioned; P300, E1A binding protein P300.

Table IV. Characteristics of the studies included in Fig. 4.

\begin{tabular}{|c|c|c|c|c|c|c|c|c|c|c|}
\hline \multirow{2}{*}{$\begin{array}{l}\text { First author } \\
\text { (year) }\end{array}$} & \multirow{2}{*}{$\begin{array}{l}\text { Cohort } \\
\text { country } \\
\text { of origin }\end{array}$} & \multirow{2}{*}{$\begin{array}{l}\text { Dominant } \\
\text { ethnicity }\end{array}$} & \multirow{2}{*}{$\begin{array}{l}\text { Cancer } \\
\text { type }\end{array}$} & \multicolumn{2}{|c|}{$\begin{array}{l}\text { P300 } \\
\text { expression } \\
\text { status }(n)\end{array}$} & \multicolumn{2}{|c|}{ PFS/RFS/DFS } & \multirow{2}{*}{$\begin{array}{l}\text { Survival } \\
\text { analysis }\end{array}$} & \multirow{2}{*}{$\begin{array}{l}\text { Maximum } \\
\text { follow-up } \\
\text { (moths) }\end{array}$} & \multirow[b]{2}{*}{ (Refs.) } \\
\hline & & & & High & Low & HR $(95 \%$ CI) & $\mathrm{P}$-value & & & \\
\hline Chen (2014) & China & Asian & CSCC & 86 & 79 & $1.78(1.08,2.93)$ & 0.024 & RFS & 120 & (15) \\
\hline Huh (2013) & Korea & Asian & $\mathrm{CRC}$ & 149 & 50 & $3.314(1.109,9.9)$ & NM & DFS & 120 & (20) \\
\hline Liao (2012) & China & Asian & NPC & 127 & 82 & $1.55(0.93,2.59)$ & NM & PFS & 250 & (21) \\
\hline Xiao (2011) & China & Asian & $\mathrm{BC}$ & 105 & 88 & $2.05(1.3,4.59)$ & 0.017 & PFS & 100 & (10) \\
\hline
\end{tabular}

CSCC, cutaneous squamous cell carcinoma; CRC, colorectal cancer; GEJC, gastroesophageal junction cancer; NPC, nasopharyngeal carcinoma; BC, breast cancer; PFS, progression-free survival; RFS, relapse-free survival; DFS, disease-free survival; OS, overall survival; NM, not mentioned; P300, E1A binding protein P300.

detailed meta-analysis comprising 2,517 cases. The results indicated that overexpression of P300 was significantly associated with poor PFS/RFS/DFS, which is consistent with results of the study by Xiao et al (10), in which there was a significant association between high expression of P300 and poor PFS. However, P300 overexpression was not significantly associated with OS.

Although P300 has been observed as a tumour suppressor in the majority of studies, its oncogenic role has also been confirmed in multiple cancer types, where it is involved in the EMT $(23,27,28)$, proliferation $(29,30)$ and apoptosis $(30,31)$. Gao et al (16) suggested that high expression of P300 enhanced EMT of HCC cells, and Liao et al (27) also indicates that P300 promotes EMT in an NPC cell line. Pifer et al (28) suggested that grainyhead-like 2 inhibits the P300 co-activator, suppressing EMT. Inagaki et al (29), indicated that core-binding protein/P300 histone acetyltransferase activity is responsible for epigenetic regulation of proliferation and invasion in HCC cells, and Dou et al (32) suggested that midazolam inhibits the proliferation of human head and neck SCC cells by downregulating P300 expression. Gao et al (30) reported that the P300 inhibitor C646 induces cell cycle arrest and apoptosis in acute myeloid leukemia cells and Ono et al (31) indicated that P300 inhibition enhances the gemcitabine-induced apoptosis of pancreatic cancer cells. 


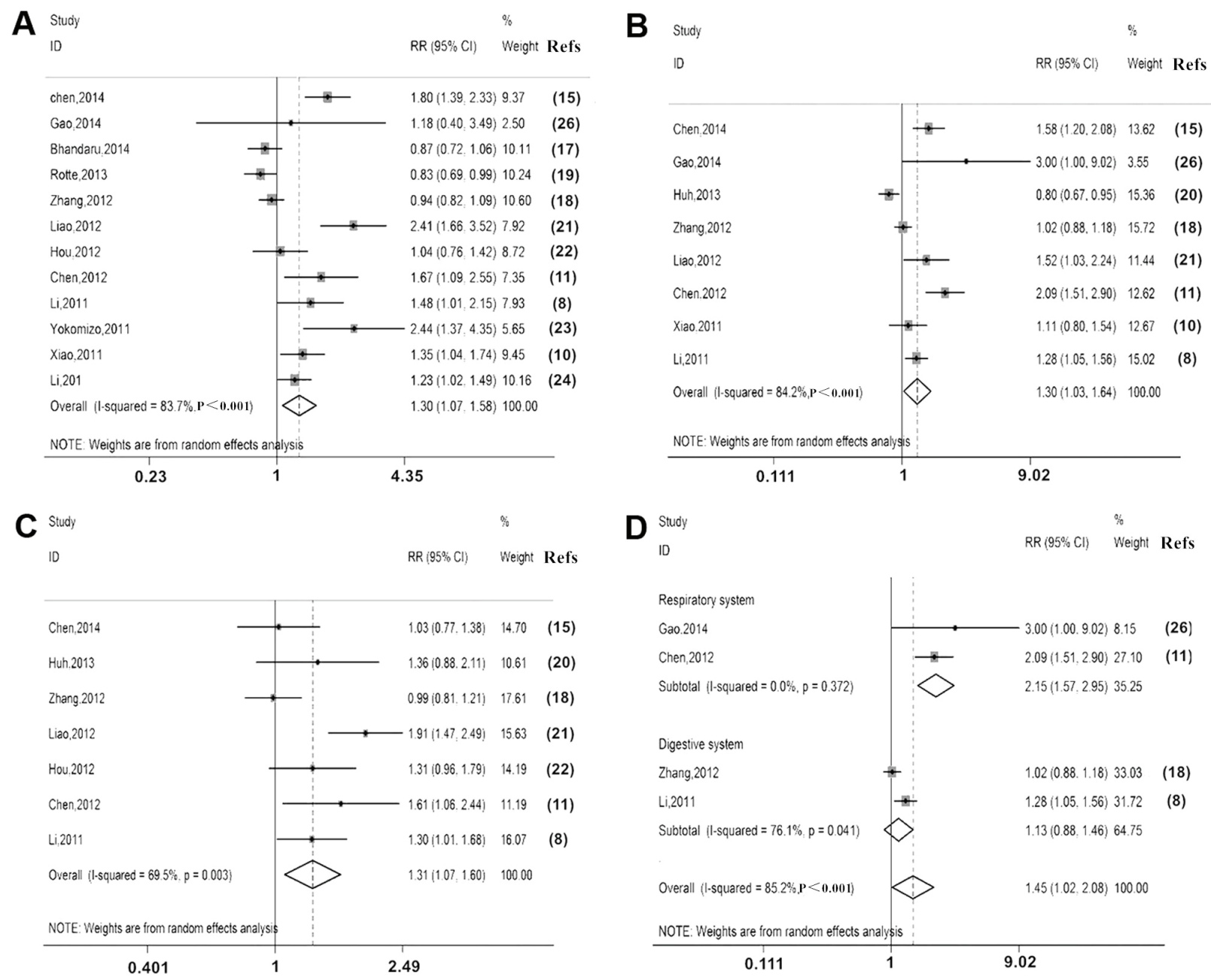

Figure 5. Forest plots for the association between E1A binding protein P300 expression and various clinicopathological parameters. (A) Clinical stage (III/IV vs. I/II). (B) Lymph node metastasis (M1 vs. M0). (C) Depth of invasion (T3/T4 vs. T1/T2). (D) Lymph node metastasis in respiratory and digestive systems (M1 vs. M0). The horizontal bar represents the confidence interval, the small square represents the HR and the diamon represents the overall HR with the confidence interval. RR, relative risk.

A

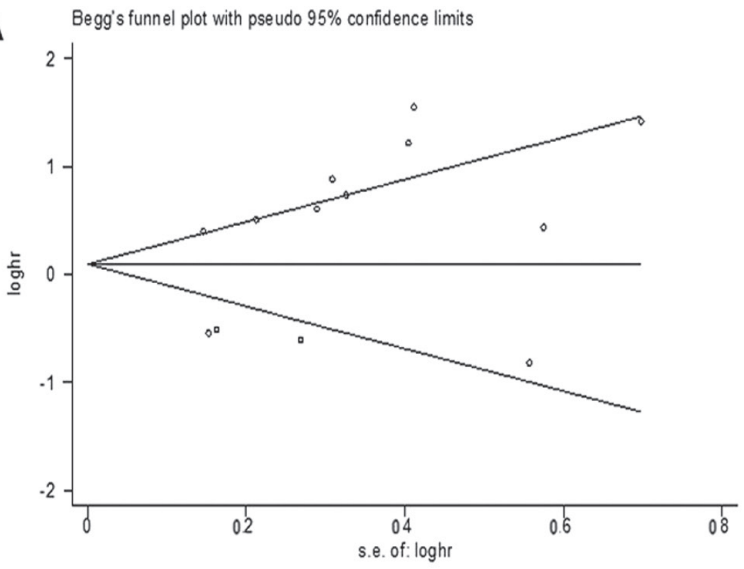

B

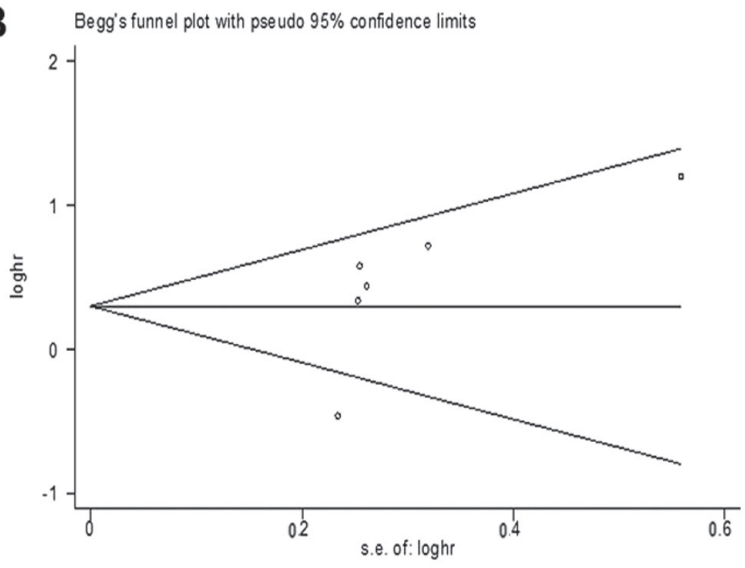

Figure 6. Begg's funnel plots for bias analysis of studies analyzing overall, progression-free, recurrence-free and disease-free survival. (A) Begg's funnel plots for overall survival. (B) Begg's funnel plots for progression-free survival, relapse-free survival and disease-free survival. S.e., standard error.

In the present study, subgroup analyses were performed based on ethnicity and location of the malignant neoplasms. The ethnicity analysis demonstrated that overexpression of P300 in Asian patients was significantly associated with poor OS. However, overexpression of P300 was a favourable predictor of OS in Caucasians. The differences between the Asian and Caucasian ethnic groups demonstrate heterogeneity, and are associated with socioeconomic status, culture, diet, environment and genetics. Biological functions, including DNA methylation, which is a type of epigenetic process, has 


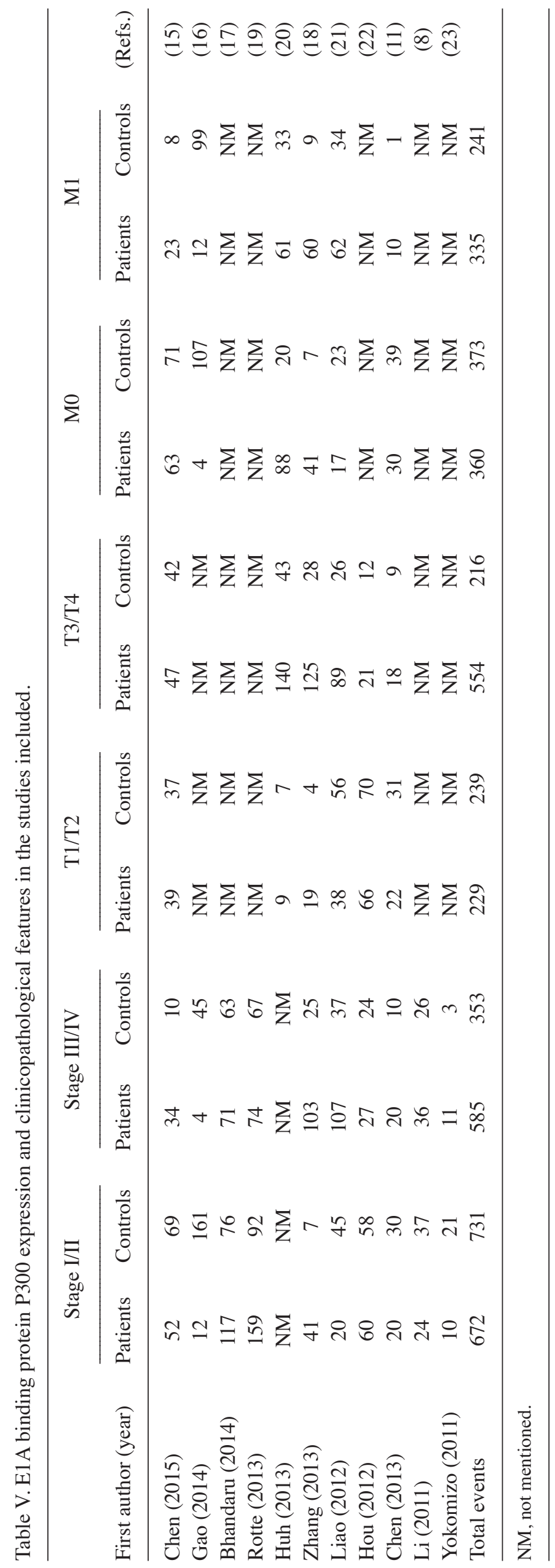


Table VI. RR for the association between clinicopathological features and high expression of E1A binding protein P300.

\begin{tabular}{|c|c|c|c|c|c|c|}
\hline \multirow{2}{*}{$\begin{array}{l}\text { Clinicopathological } \\
\text { feature }\end{array}$} & \multirow{2}{*}{$\begin{array}{l}\text { Number of } \\
\text { studies }\end{array}$} & \multirow[b]{2}{*}{ Cases (n) } & \multirow{2}{*}{$\begin{array}{l}\text { Analytical } \\
\text { model }\end{array}$} & \multirow{2}{*}{$\begin{array}{r}\text { Pooled RR } \\
(95 \% \mathrm{CI})\end{array}$} & \multicolumn{2}{|c|}{ Heterogeneity } \\
\hline & & & & & $\mathrm{I}^{2}(\%)$ & P-value \\
\hline Tumor size ( $\leq 5$ vs. $>5 \mathrm{~cm})$ & 4 & 663 & Random-effects model & $0.89(0.67,1.19)$ & 86.6 & $<0.001$ \\
\hline $\begin{array}{l}\text { Degree of differentiation } \\
\text { (well + moderate vs. poor) }\end{array}$ & 9 & 1,371 & Random-effects model & $0.86(0.71,1.04)$ & 72.7 & $<0.001$ \\
\hline Gender (female vs. male) & 11 & 2,019 & Fixed-effects model & $0.97(0.90,1.06)$ & 0 & 0.849 \\
\hline
\end{tabular}

$\mathrm{RR}$, relative risk.

a major role in the induction of genetics. DNA methylation may cause changes of the key regulatory genes in cancer. Genes that are differentially methylated, as observed between Asian and Caucasian populations, are involved in oncogenic processes, including tumour growth, tumour suppression and metastasis (33). Langevin and Kelsey (34) also suggested that the oncogenic process is driven by the accumulation of genetic and epigenetic alterations, resulting in dysregulation of tumour suppressor genes, key oncogenes and DNA repair genes.

In the present study, P300 was associated with poor OS of Asians with malignant neoplasms of the digestive system. The present results are consistent with those reported by McCracken et al (35), who reported that compared to Caucasians, Asians are more affected by certain cancer types, including those of the stomach, liver, oesophagus and the uterine cervix. Chien et al (36), also demonstrated that 10-60\% of Asian Americans (Chinese and Mexican men), are likely to be diagnosed with stage III or IV CRC compared to Caucasian men.

The association between P300 expression and clinicopathological parameters was also analysed in the present study. The results indicated that overexpression of P300 was associated with clinical stage, lymph node metastasis and depth of invasion. However, it was not significantly associated with sex, tumour size or degree of differentiation. The present results are consistent with those of the study by Liao et al (21), who indicated that overexpression of P300 was positively associated with later T classification, later $\mathrm{N}$ classification, distant metastasis and later clinical stage. Xiao et al (10) also suggested that high expression of P300 was positively correlated with higher histological grade, advanced clinical stage and tumour recurrence. However, the present results did not indicate any significant association of P300 with those clinicopathological parameters.

It is important to consider the limitations of the present meta-analysis. First, it was only possible to directly extract the data from 9 of the 14 eligible studies, while the data of the remaining studies were extracted from Kaplan-Meier survival plots. Second, the cut-off values of P300 expression in the original studies were varied, e.g. the mean values were used in certain studies, while the median values were used in the others. Third, some data points within the funnel plot are located outside the funnel. Furthermore, as tissue was kept for many years, it is possible that protein may be subject to decomposition after long periods of storage. Furthermore, heterogeneity existed in the total analysis of OS $\left(\mathrm{I}^{2}=86.2 \%\right)$ and PFS/RFS/DFS $\left(\mathrm{I}^{2}=69.6 \%\right)$. This is likely due to the different ethnicities, types of malignant cancer, detection methods and follow-up periods. These factors should be considered when evaluating the prognostic role of P300 expression in human malignant cancers in the future. If these limitations were the be overcome, P300 may be used as a prognostic biomarker in clinical applications.

In conclusion, the present results indicated that high P300 expression predicted a poor OS in Asian populations, particularly in digestive system malignant neoplasms. However, more comprehensive studies are required to evaluate and confirm the prognostic value of $\mathrm{P} 300$ in cancer.

\section{Acknowledgements}

Not applicable.

\section{Funding}

The present study was supported by grants from the National Natural Science Foundation of China (grant no. 81860471), the Outstanding Youth Science and Technology Talent Cultivation Plan of Shihezi University (grant no. 2015ZRKXJQ07), the International cooperation projects of Shihezi University (grant no. GJHZ201710) and the Research Project of High-Level Talents of Shihezi University (grant no. RCZX201549).

\section{Availability of data and materials}

All data are available from the corresponding author on reasonable request.

\section{Authors' contributions}

XL, HZ, YH and NW performed the experiments and analyzed the data; JH, XC, JZ and YQ designed and supervised the study. WZ, WG and LP provided crucial input for the project; ZL and YH wrote the manuscript. All authors read and approved the final version of the manuscript.

\section{Ethics approval and informed consent}

The present study was approved by the Institutional Ethics Review Board (IERB; no. 2013LL10) at the First Affiliated Hospital, Shihezi University School of Medicine (Shihezi, China; including the use of patients' tissues between 1968 and 2015). 


\section{Patients consent for publication}

Not applicable.

\section{Competing interests}

All authors declare that they have no competing interests.

\section{References}

1. Kundu TK, Palhan VB, Wang Z, An W, Cole PA and Roeder RG: Activator-dependent transcription from chromatin in vitro involving targeted histone acetylation by p300. Mol Cell 6: 551-561, 2000.

2. Vo N and Goodman RH: CREB-binding protein and p300 in transcriptional regulation. J Biol Chem 276: 13505-13508, 2001.

3. Huang WC and Chen CC: Akt phosphorylation of p300 at Ser-1834 is essential for its histone acetyltransferase and transcriptional activity. Mol Cell Biol 25: 6592-6602, 2005

4. Muraoka M,Konishi M,Kikuchi-Yanoshita R, Tanaka K, Shitara N, Chong JM, Iwama T and Miyaki M: p300 gene alterations in colorectal and gastric carcinomas. Oncogene 12: 1565-1569, 1996.

5. Wang L, Huang X, Chai Y, Zou L, Chedrawe M and Ding Y: Octreotide inhibits the proliferation of gastric cancer cells through P300-HAT activity and the interaction of ZAC and P300. Oncol Rep 37: 2041-2048, 2017.

6. Gayther SA, Batley SJ, Linger L, Bannister A, Thorpe K, Chin SF, Daigo Y, Russell P, Wilson A, Sowter HM, et al: Mutations truncating the EP300 acetylase in human cancers. Nat Genet 24: 300-303, 2000

7. Shikama N, Lee CW, France S, Delavaine L, Lyon J, Krstic-Demonacos M and La Thangue NB: A novel cofactor for p300 that regulates the p53 response. Mol Cell 4: 365-376, 1999.

8. Li M, Luo RZ, Chen JW, Cao Y, Lu JB, He JH, Wu QL and Cai MY: High expression of transcriptional coactivator p300 correlates with aggressive features and poor prognosis of hepatocellular carcinoma. J Transl Med 9: 5, 2011.

9. Isharwal SM, Miller MC, Marlow C, Makarov DV, Partin AW and Veltri RW: p300 (histone acetyltransferase) biomarker predicts prostate cancer biochemical recurrence and correlates with changes in epithelia nuclear size and shape. Prostate 68: 1097-1104, 2008.

10. Xiao XS, Cai MY, Chen JW, Guan XY, Kung HF, Zeng YX and Xie D: High expression of p300 in human breast cancer correlates with tumor recurrence and predicts adverse prognosis. Chin J Cancer Res 23: 201-207, 2011.

11. Chen YF, Luo RZ, Li Y, Cui BK, Song M, Yang AK and Chen WK: High expression levels of COX-2 and P300 are associated with unfavorable survival in laryngeal squamous cell carcinoma. Eur Arch Otorhinolaryngol 270: 1009-1017, 2013

12. Qi Y, Wang CC, He YL, Zou H, Liu CX, Pang LJ, Hu JM, Jiang JF, Zhang WJ and Li F: The correlation between morphology and the expression of TGF- $\beta$ signaling pathway proteins and epithelial-mesenchymal transition-related proteins in synovial sarcomas. Int J Clin Exp Pathol 6: 2787-2799, 2013.

13. Greenwood DC: Meta-analysis of observational studies. Mod Methods Epidemiol: 173-189, 2012.

14. Egger M, Davey Smith G, Schneider M and Minder C: Bias in meta-analysis detected by a simple, graphical test. BMJ 315: 629-634, 1997.

15. Chen MK, Cai MY, Luo RZ, Tian X, Liao QM, Zhang XY and Han JD: Overexpression of p300 correlates with poor prognosis in patients with cutaneous squamous cell carcinoma. $\mathrm{Br}$ Dermatol 172: 111-119, 2015.

16. Gao Y, Geng J, Hong X, Qi J, Teng Y, Yang Y, Qu D and Chen G: Expression of p300 and CBP is associated with poor prognosis in small cell lung cancer. Int J Clin Exp Pathol 7: 760-767, 2014.

17. Bhandaru M, Ardekani GS, Zhang G, Martinka M, McElwee KJ, $\mathrm{Li} \mathrm{G}$ and Rotte A: A combination of $\mathrm{p} 300$ and Braf expression in the diagnosis and prognosis of melanoma. BMC Cancer 14: 398, 2014.

18. Zhang LH, Huang Q, Fan XS, Wu HY, Yang J and Feng AN: Clinicopathological significance of SIRT1 and p300/CBP expression in gastroesophageal junction (GEJ) cancer and the correlation with E-cadherin and MLH1. Pathol Res Pract 209: 611-617, 2013.
19. Rotte A, Bhandaru M, Cheng Y, Sjoestroem C, Martinka M and Li G: Decreased expression of nuclear p300 is associated with disease progression and worse prognosis of melanoma patients. PLoS One 8: e75405, 2013.

20. Huh JW, Kim HC, Kim SH, Park YA, Cho YB, Yun SH, Lee WY and Chun H: Prognostic impact of p300 expression in patients with colorectal cancer. J Surg Oncol 108: 374-377, 2013

21. Liao ZW, Zhou TC, Tan XJ, Song XL, Liu Y, Shi XY, Huang WJ, Du LL, Tu BJ and Lin XD: High expression of p300 is linked to aggressive features and poor prognosis of nasopharyngeal carcinoma. J Transl Med 10: 110, 2012

22. Ono H, Basson MD and Ito H: P300 inhibition enhances gemcitabine-induced apoptosis of pancreatic cancer. Oncotarget 7: 51301-51310, 2016.

23. Hou X, Li Y, Luo RZ, Fu JH, Zhang LJ and Yang HX: High expression of the transcriptional co-activator $\mathrm{p} 300$ predicts poor survival in resectable non-small cell lung cancers. Eur J Surg Oncol 38: 523-530, 2012

24. Li Y, Yang HX, Luo RZ, Zhang Y, Li M, Wang X and Jia WH: High expression of p300 has an unfavorable impact on survival in resectable esophageal squamous cell carcinoma. Ann Thorac Surg 91: 1531-1538, 2011.

25. Ishihama K, Yamakawa M, Semba S, Takeda H, Kawata S, Kimura S and Kimura W: Expression of HDAC1 and CBP/p300 in human colorectal carcinomas. J Clin Pathol 60: 1205-1210, 2007.

26. Qi Y, Wang N, Pang LJ, Zou H, Hu JM, Zhao J, Zhang J, Liu CX, Zhang WJ, Yuan XL and Li F: Identification of potential mutations and genomic alterations in the epithelial and spindle cell components of biphasic synovial sarcomas using a human exome SNP chip. BMC Med Genomics 8: 69, 2015.

27. Liao ZW, Zhao L, Cai MY, Xi M, He LR, Yu F, Zhou TC and Liu MZ: P300 promotes migration, invasion and epithelial-mesenchymal transition in a nasopharyngeal carcinoma cell line. Oncol Lett 13: 763-769, 2017.

28. Pifer PM, Farris JC, Thomas AL, Stoilov P, Denvir J, Smith DM and Frisch SM: Grainyhead-like 2 inhibits the coactivator p300, suppressing tubulogenesis and the epithelial-mesenchymal transition. Mol Biol Cell 27: 2479-2492, 2016.

29. Inagaki Y, Shiraki K, Sugimoto K, Yada T, Tameda M, Ogura S, Yamamoto N, Takei Y and Ito M: Epigenetic regulation of proliferation and invasion in hepatocellular carcinoma cells by $\mathrm{CBP} / \mathrm{p} 300$ histone acetyltransferase activity. Int J Oncol 48: 533-540, 2016.

30. Gao XN, Lin J, Ning QY, Gao L, Yao YS, Zhou JH, Li YH, Wang LL and Yu L: A histone acetyltransferase p300 inhibitor C646 induces cell cycle arrest and apoptosis selectively in AML1-ETO-positive AML cells. PLoS One 8: e55481, 2013.

31. Ono H, Basson MD and Ito H: P300 inhibition enhances gemcitabine-induced apoptosis of pancreatic cancer. Oncotarget 7: 51301-51310, 2016.

32. Dou YL, Lin JP, Liu FE, Wang LY, Shu HH, Jiang N, Xie Y and Duan Q: Midazolam inhibits the proliferation of human head and neck sqamous carcinoma cells by downregulating p300 expression. Tumour Biol 35: 7499-7504, 2014.

33. Mohammed SI, Springfield S and Das R: Role of epigenetics in cancer health disparities. Methods Mol Biol 863: 395-410, 2012.

34. Langevin SM and Kelsey KT: The fate is not always written in the genes: Epigenomics in epidemiologic studies. Environ Mol Mutagen 54: 533-541, 2013.

35. McCracken M, Olsen M, Chen MS Jr, Jemal A, Thun M, Cokkinides V, Deapen D and Ward E: Cancer incidence, mortality, and associated risk factors among Asian Americans of Chinese, Filipino, Vietnamese, Korean, and Japanese ethnicities. CA Cancer J Clin 57: 190-205, 2007.

36. Chien C, Morimoto LM, Tom J and Li CI: Differences in colorectal carcinoma stage and survival by race and ethnicity. Cancer 104: 629-639, 2005. 